\title{
Global behavior of 1D compressible isentropic Navier-Stokes equations with a non-autonomous external force
}

Lan Huang ${ }^{*}$ and Ruxu Lian

\begin{tabular}{l}
\hline * Correspondence: \\
huanglan82@hotmail.com \\
College of Mathematics and \\
Information Science, North China \\
University of Water Sources and \\
Electric Power, Zhengzhou 450011, \\
People's Republic of PR China
\end{tabular}

* Correspondence: anglan82@hotmail.con College of Mathematics an Information Science, North China Electric Power, Zhengzhou 450011

\begin{abstract}
In this paper, we study a free boundary problem for compressible Navier-Stokes equations with density-dependent viscosity and a non-autonomous external force. The viscosity coefficient $\mu$ is proportional to $\rho^{\theta}$ with $0<\theta<1$, where $\rho$ is the density. Under certain assumptions imposed on the initial data and external force $f$, we obtain the global existence and regularity. Some ideas and more delicate estimates are introduced to prove these results.
\end{abstract}

Keywords: Compressible Navier-Stokes equations, Viscosity, Regularity, Vacuum

\section{Introduction}

We study a free boundary problem for compressible Navier-Stokes equations with density-dependent viscosity and a non-autonomous external force, which can be written in Eulerian coordinates as:

$$
\begin{aligned}
& \rho_{\tau}+(\rho u)_{\xi}=0, \quad \tau>0 \\
& (\rho u)_{\tau}+\left(\rho u^{2}+P(\rho)\right)_{\xi}=\left(\mu u_{\xi}\right)_{\xi}+\rho f, \quad a(\tau)<\xi<b(\tau)
\end{aligned}
$$

with initial data

$$
(\rho, u)(\xi, 0)=\left(\rho_{0}, u_{0}\right)(\xi), \quad a=a(0) \leq \xi \leq b(0)=b,
$$

where $\rho=\rho(\xi, \tau), u=u(\xi, \tau), P=P(\rho)$ and $f=f(\xi, t)$ denote the density, velocity, pressure and a given external force, respectively, $\mu=\mu(\rho)$ is the viscosity coefficient. $a(\tau)$ and $b(\tau)$ are the free boundaries with the following property:

$$
\begin{aligned}
& \frac{\mathrm{d}}{\mathrm{d} \tau} a(\tau)=u(a(\tau), \tau), \quad \frac{\mathrm{d}}{\mathrm{d} \tau} b(\tau)=u(b(\tau), \tau), \\
& \left(-P(\rho)+\mu(\rho) u_{\xi}\right)(\xi, \tau)=0, \quad \xi=a(\tau), b(\tau) .
\end{aligned}
$$

The investigation in [1] showed that the continuous dependence on the initial data of the solutions to the compressible Navier-Stokes equations with vacuum failed. The main reason for the failure at the vacuum is because of kinematic viscosity coefficient being independent of the density. On the other hand, we know that the Navier-Stokes equations can be derived from the Boltzmann equation through Chapman-Enskog

\section{至

(C) 2011 Huang and Lian; licensee Springer. This is an Open Access article distributed under the terms of the Creative Commons Attribution License (http://creativecommons.org/licenses/by/2.0), which permits unrestricted use, distribution, and reproduction in any medium, provided the original work is properly cited. 
expansion to the second order, and the viscosity coefficient is a function of temperature. For the hard sphere model, it is proportional to the square-root of the temperature. If we consider the isentropic gas flow, this dependence is reduced to the dependence on the density function by using the second law of thermal dynamics.

For simplicity of presentation, we consider only the polytropic gas, i.e. $P(\rho)=A \rho^{\gamma}$ with $A>0$ being constants. Our main assumption is that the viscosity coefficient $\mu$ is assumed to be a functional of the density $\rho$, i.e. $\mu=c \rho^{\theta}$, where $c$ and $\theta$ are positive constants. Without loss of generality, we assume $A=1$ and $c=1$.

Since the boundaries $x=a(\tau)$ and $x=b(\tau)$ are unknown in Euler coordinates, we will convert them to fixed boundaries by using Lagrangian coordinates. We introduce the following coordinate transformation

$$
x=\int_{a(\tau)}^{\xi} \rho(y, \tau) \mathrm{d} y, \quad t=\tau,
$$

then the free boundaries $\xi=a(\tau)$ and $\xi=b(\tau)$ become

$$
x=0, \quad x=\int_{a(\tau)}^{b(\tau)} \rho(z, \tau) \mathrm{d} z=\int_{a}^{b} \rho_{0}(z) \mathrm{d} z
$$

where $\int_{a}^{b} \rho_{0}(z) \mathrm{d} z$ is the total initial mass, and without loss of generality, we can normalize it to 1 . So in terms of Lagrangian coordinates, the free boundaries become fixed. Under the coordinate transformation, Eqs. (1.1)-(1.2) are now transformed into

$$
\begin{aligned}
& \rho_{t}+\rho^{2} u_{x}=0, \quad t>0, \\
& u_{t}+P(\rho)_{x}=\left(\rho \mu(\rho) u_{x}\right)_{x}+f(r, t), \quad 0<x<1
\end{aligned}
$$

where $r=\int_{0}^{x} \rho^{-1}(y, t) \mathrm{d} y$. The boundary conditions (1.4)-(1.5) become

$$
\left(-\rho^{\gamma}+\rho^{1+\theta} u_{x}\right)(d, t)=0, \quad d=0,1,
$$

and the initial data (1.3) become

$$
(\rho, u)(x, 0)=\left(\rho_{0}, u_{0}\right)(x), \quad x \in[0,1] .
$$

Now let us first recall some previous works in this direction. When the external force $f \equiv 0$, there have been many works (see, e.g., [2-9]) on the existence and uniqueness of global weak solutions, based on the assumption that the gas connects to vacuum with jump discontinuities, and the density of the gas has compact support. Among them, Liu et al. [4] established the local well-posedness of weak solutions to the Navier-Stokes equations; Okada et al. [5] obtained the global existence of weak solutions when $0<\theta<1 / 3$ with the same property. This result was later generalized to the case when $0<\theta<1 / 2$ and $0<\theta<1$ by Yang et al. [7] and Jiang et al. [3], respectively. Later on, Qin et al. [8,9] proved the regularity of weak solutions and existence of classical solution. Fang and Zhang [2] proved the global existence of weak solutions 
to the compressible Navier-Stokes equations when the initial density is a piece-wise smooth function, having only a finite number of jump discontinuities.

For the related degenerated density function and viscosity coefficient at free boundaries, see Yang and Zhao [10], Yang and Zhu [11], Vong et al. [12], Fang and Zhang $[13,14]$, Qin et al. [15], authors studied the global existence and uniqueness under some assumptions on initial data.

When $f \neq 0$, Qin and Zhao [16] proved the global existence and asymptotic behavior for $\gamma=1$ and $\mu$ const with boundary conditions $u(0, t)=u(1, t)=0$; Zhang and Fang [17] established the global behavior of the Equations (1.1)-(1.2) with boundary conditions $u(0, t)=\rho(1, t)=0$. In this paper, we obtain the global existence of the weak solutions and regularity with boundary conditions (1.4)-(1.5). In order to obtain existence and higher regularity of global solutions, there are many complicated estimates on external force and higher derivations of solution to be involved, this is our difficulty. To overcome this difficulty, we should use some proper embedding theorems, the interpolation techniques as well as many delicate estimates. This is the novelty of the paper.

The notation in this paper will be as follows:

$L^{p}, 1 \leq p \leq+\infty, W^{m, p}, m \in N, H^{1}=W^{1,2}, H_{0}^{1}=W_{0}^{1,2}$ denote the usual (Sobolev) spaces on $[0,1]$. In addition, $\|\cdot\|_{B}$ denotes the norm in the space $B$; we also put $\|\cdot\|=\|\cdot\|_{L^{2}([0,1])}$.

The rest of this paper is organized as follows. In Section 2, we shall prove the global existence in $H^{1}$. In Section 3, we shall establish the global existence in $H^{2}$. In Section 4, we give the detailed proof of Theorem 4.1.

\section{Global existence of solutions in $H^{1}$}

In this section, we shall establish the global existence of solutions in $H^{1}$.

Theorem 2.1 Let $0<\theta<1, \gamma>1$, and assume that the initial data $\left(\rho_{0}, u_{0}\right)$ satisfies $\inf _{[0,1]} \rho_{0}>0, \rho_{0} \in W^{1,2 n}, u_{0} \in H^{1}$ and external force $f$ satisfies $f(r(x, \cdot), \cdot) \in L^{2 n}\left([0, T], L^{2 n}\right.$ $[0,1])$ for some $n \in N$ satisfying $n(2 n-1) /\left(2 n^{2}+2 n-1\right)>\theta$, then there exists a unique global solution $(\rho(x, t), u(x, t))$ to problem (1.8)-(1.11), such that for any $T>0$,

$$
\begin{aligned}
& 0<C_{1}^{-1}(T) \leq \rho(x, t) \leq C_{1}(T), \quad \rho \in L^{\infty}\left([0, T], H^{1}[0,1]\right), \\
& u \in L^{\infty}\left([0, T], H^{1}[0,1]\right) \cap \in L^{2}\left([0, T], H^{2}[0,1]\right), \quad u_{t} \in L^{2}\left([0, T], L^{2}[0,1]\right) .
\end{aligned}
$$

The proof of Theorem 2.1 can be done by a series of lemmas as follows.

Lemma 2.1 Under conditions of Theorem 2.1, the following estimates hold

$$
\begin{aligned}
& \int_{0}^{1}\left(\frac{1}{2} u^{2}+\frac{1}{\gamma-1} \rho^{\gamma-1}\right) \mathrm{d} x+\int_{0}^{t} \int_{0}^{1} \rho^{1+\theta} u_{x}^{2}(x, s) \mathrm{d} x \mathrm{~d} s \leq C_{1}(T), \\
& \rho(x, t) \leq C_{1}(T), \quad(x, t) \in[0,1] \times[0, T] \\
& \int_{0}^{1} u^{2 n} \mathrm{~d} x+n(2 n-1) \int_{0}^{t} \int_{0}^{1} \rho^{1+\theta} u^{2 n-2} u_{x}^{2}(x, s) \mathrm{d} x \mathrm{~d} s \leq C_{1}(T)
\end{aligned}
$$


where $C_{1}(T)$ denotes generic positive constant depending only on $\left\|\rho_{0}\right\|_{W^{1,2 n}[0,1]},\left\|u_{0}\right\|_{H^{1}[0,1]}$, time $T$ and $\|f\|_{L^{2 n}}\left([0, T], L^{2 n}[0,1]\right)$.

Proof Multiplying (1.8) and (1.9) by $\rho^{\gamma-2}$ and $u$, respectively, using integration by parts, and considering the boundary conditions (1.10), we have

$$
\frac{\mathrm{d}}{\mathrm{d} t} \int_{0}^{1}\left(\frac{1}{2} u^{2}+\frac{1}{\gamma-1} \rho^{\gamma-1}\right) \mathrm{d} x+\int_{0}^{1} \rho^{1+\theta} u_{x}^{2} \mathrm{~d} x=\int_{0}^{1} f u \mathrm{~d} x
$$

Integrating (2.4) with respect to $t$ over $[0, t]$, using Young's inequality, we have

$$
\begin{aligned}
\int_{0}^{1}\left(\frac{1}{2} u^{2}+\frac{1}{\gamma-1} \rho^{\gamma-1}\right) \mathrm{d} x+\int_{0}^{t} \int_{0}^{1} \rho^{1+\theta} u_{x}^{2} \mathrm{~d} x \mathrm{~d} s & \leq C_{1}(T)+\frac{1}{2} \int_{0}^{t} \int_{0}^{1} u^{2} \mathrm{~d} x+C_{1} \int_{0}^{t} \int_{0}^{1} f^{2} \mathrm{~d} x \mathrm{~d} s \\
& \leq \frac{1}{2} \int_{0}^{t} \int_{0}^{1} u^{2} \mathrm{~d} x+C_{1}(T)
\end{aligned}
$$

which, by virtue of Gronwall's inequality and assumption $f(r(x, \cdot), \cdot) \in L^{2 n}\left([0, T], L^{2 n}\right.$ $[0,1])$, gives $(2.1)$.

We derive from (1.8) that

$$
\left(\rho^{\theta}\right)_{t}=-\theta \rho^{1+\theta} u_{x}
$$

Integrating (2.5) with respect to $t$ over $[0, t]$ yields

$$
\rho^{\theta}(x, t)=\rho_{0}^{\theta}-\theta \int_{0}^{t} \rho^{1+\theta} u_{x}(x, s) \mathrm{d} s
$$

Integrating (1.9) with respect to $x$, applying the boundary conditions (1.10), we obtain

$$
\rho^{1+\theta} u_{x}=\int_{0}^{x} u_{t} \mathrm{~d} y+\rho^{\gamma}-\int_{0}^{x} f(r(y, t), t) \mathrm{d} y
$$

Inserting (2.7) into (2.6) gives

$$
\rho^{\theta}+\theta \int_{0}^{t} \rho^{\gamma} \mathrm{d} s=\rho_{0}^{\theta}+\theta \int_{0}^{t} \int_{0}^{x} f(r,(y, s), s) \mathrm{d} y \mathrm{~d} s-\theta \int_{0}^{x}\left(u-u_{0}\right) \mathrm{d} y
$$

Thus, the Hölder inequality and (2.1) imply

$$
\left|\int_{0}^{x} u(y, t) \mathrm{d} y\right| \leq C_{1}
$$

and (2.2) follows from (2.8) and (2.9). 
Multiplying (1.9) by $2 n u^{2 n-1}$ and integrating over $x$ and $t$, applying the boundary conditions (1.10), we have

$$
\begin{aligned}
& \int_{0}^{1} u^{2 n} \mathrm{~d} x+2 n(2 n-1) \int_{0}^{t} \int_{0}^{1} u^{2 n-2} \rho^{1+\theta} u_{x}^{2} \mathrm{~d} x \mathrm{~d} s \\
& \quad=\int_{0}^{1} u_{0}^{2 n} \mathrm{~d} x+2 n(2 n-1) \int_{0}^{t} \int_{0}^{1} u^{2 n-2} \rho^{\gamma} u_{x} \mathrm{~d} x \mathrm{~d} s+2 n \int_{0}^{t} \int_{0}^{1} f u^{2 n-1} \mathrm{~d} x \mathrm{~d} s .
\end{aligned}
$$

Applying the Young inequality and condition $f(r(x, \cdot), \cdot) \in L^{2 n}\left([0, T], L^{2 n}[0,1]\right)$ to the last two terms in (2.10) yields

$$
\begin{aligned}
& \int_{0}^{1} u^{2 n} \mathrm{~d} x+n(2 n-1) \int_{0}^{t} \int_{0}^{1} u^{2 n-2} \rho^{1+\theta} u_{x}^{2} \mathrm{~d} x \mathrm{~d} s \\
& \leq C_{1}+\int_{0}^{t} \int_{0}^{1} f^{2 n} \mathrm{~d} x \mathrm{~d} s+(2 n-1) \int_{0}^{t} \int_{0}^{1} u^{2 n} \mathrm{~d} x \mathrm{~d} s \\
& \quad+n(2 n-1) \int_{0}^{t} \int_{0}^{1} u^{2 n-2} \rho^{2 \gamma-1-\theta} \mathrm{d} x \mathrm{~d} s \\
& \leq C_{1}(T)+n(2 n-1) \int_{0}^{t} \int_{0}^{1}\left(\frac{1}{n} \rho^{(2 \gamma-1-\theta) n}+\frac{n-1}{n} u^{2 n}\right) \mathrm{d} x \mathrm{~d} s+(2 n-1) \int_{0}^{t} \int_{0}^{1} u^{2 n} \mathrm{~d} x \mathrm{~d} s \\
& \leq C_{1}(T)+n(2 n-1) \int_{0}^{1} \int_{0}^{2 n} u^{2 n} \mathrm{~d} x \mathrm{~d} s .
\end{aligned}
$$

Applying Gronwall's inequality, we conclude

$$
\int_{0}^{1} u^{2 n} \mathrm{~d} x \leq C_{1}(T)
$$

, which, along with (2.11), yields (2.3). The proof of Lemma 2.1 is complete.

Lemma 2.2 Under conditions of Theorem 2.1, the following estimates hold

$$
\begin{aligned}
& \int_{0}^{1}\left(\rho^{\theta}\right)_{x}^{2 n} \mathrm{~d} x \leq C_{1}(T), \\
& \rho(x, t) \geq C_{1}^{-1}(T)>0 .
\end{aligned}
$$

Proof We derive from (2.5) and (1.9) that

$$
\left(\rho^{\theta}\right)_{x t}=-\theta\left(u t+\left(\rho^{\gamma}\right)_{x}-f\right) .
$$

Integrating it with respect to $t$ over $[0, t]$, we obtain

$$
\left(\rho^{\theta}\right)_{x}=\left(\rho_{0}^{\theta}\right)_{x}-\theta\left(u-u_{0}\right)-\theta \int_{0}^{t}\left(\rho^{\gamma}\right)_{x} \mathrm{~d} s+\theta \int_{0}^{t} f \mathrm{~d} s .
$$


Multiplying (2.16) by $\left[\left(\rho^{\theta}\right)_{x}\right]^{2 n-1}$, and integrating the resultant with respect to $x$ to get

$$
\begin{gathered}
\int_{0}^{1}\left(\rho^{\theta}\right)_{x}^{2 n} \mathrm{~d} x=\int_{0}^{1}\left(\rho^{\theta}\right)_{x}^{2 n-1}\left(\rho_{0}^{\theta}\right)_{x} \mathrm{~d} x \\
-\theta \int_{0}^{1}\left[\left(u-u_{0}\right)+\int_{0}^{t}\left(\rho^{\gamma}\right)_{x} \mathrm{~d} s-\int_{0}^{t} f \mathrm{~d} s\right]\left(\rho^{\theta}\right)_{x}^{2 n-1} \mathrm{~d} x \\
\leq C\left(\int_{0}^{1}\left(\rho^{\theta}\right)_{x}^{2 n} \mathrm{~d} x\right)^{\frac{2 n-1}{2 n}}\left\{\int_{0}^{1}\left(\rho_{0}^{\theta}\right)_{x}^{2 n} \mathrm{~d} x\right)^{\frac{1}{2 n}} \\
\left.+\left\|u-u_{0}\right\|_{L^{2 n}}+\int_{0}^{1}\left(\int_{0}^{t}\left(\rho^{\gamma}\right)_{x} \mathrm{~d} s\right)^{2 n} \mathrm{~d} x\right)^{\frac{1}{2 n}} \\
\left.\left.+\left(\int_{0}^{1}\left(\int_{0}^{t} f \mathrm{~d} s\right)^{2 n} \mathrm{~d} x\right)^{\frac{1}{2 n}} f^{2 n} \mathrm{~d} x\right)^{\frac{1}{2 n}} \mathrm{~d} s\right\} \\
+\left\|u-u_{0}\right\|_{L^{2 n}}+\int_{0}^{t}\left(\int_{0}^{1}\left(\rho^{\theta}\right)_{x}^{2 n} \mathrm{~d} x\right)_{0}^{\frac{2 n-1}{2 n}}\left(\int_{0}^{1}\left(\rho_{0}^{\theta}\right)_{x}^{2 n} \mathrm{~d} x\right)^{\left.\frac{1}{2 n} \mathrm{~d} s\right)^{\frac{1}{2 n}}} \mathrm{~d} x
\end{gathered}
$$

here, we use the inequality $\left\|\int g(\cdot, s)\right\|_{L^{p}} \leq \int\|g(\cdot, s)\|_{L^{p}} \mathrm{~d} s$. Using Young's inequality and assumptions of external of $f$, we get from (2.17) that

$$
\begin{aligned}
\int_{0}^{1}\left(\rho^{\theta}\right)_{x}^{2 n} \mathrm{~d} x \leq & \frac{1}{2} \int_{0}^{1}\left(\rho^{\theta}\right) \frac{2 n}{x} \mathrm{~d} x \\
& +C \int_{0}^{t} \int_{0}^{1}\left(\rho^{\gamma}\right)_{x}^{2 n} \mathrm{~d} x \mathrm{~d} s+C \int_{0}^{t} \int_{0}^{1} f^{2 n} \mathrm{~d} x \mathrm{~d} s+C_{1}(T) \\
\leq & \frac{1}{2} \int_{0}^{1}\left(\rho^{\theta}\right)_{x}^{2 n} \mathrm{~d} x+C_{1}(T) \int_{0}^{t} \int_{0}^{1}\left(\rho^{\gamma}\right)_{x}^{2 n} \mathrm{~d} x \mathrm{~d} s+C 1(T) .
\end{aligned}
$$


Hence,

$$
\int_{0}^{1}\left(\rho^{\theta}\right)_{x}^{2 n} \mathrm{~d} x \leq C 1(T)+C 1(T) \int_{0}^{t} \int_{0}^{1}\left(\rho^{\gamma}\right)_{x}^{2 n} \mathrm{~d} x \mathrm{~d} s
$$

Using the Gronwall inequality to (2.18), we obtain (2.13).

The proof of (2.14) can be found in [3], please refer to Lemma 2.3 in [3] for detail.

Lemma 2.3 Under the assumptions in Theorem 2.1, for any $0 \leq t \leq T$, we have the following estimate

$$
\left\|u_{x}(t)\right\|^{2}+\int_{0}^{t}\left\|u_{t}(s)\right\|^{2} \mathrm{~d} s \leq C_{1}(T)
$$

Proof Multiplying (1.9) by $u_{t}$, then integrating over $[0,1] \times[0, t]$, we obtain

$$
\int_{0}^{t} \int_{0}^{1} u_{t}^{2} \mathrm{~d} x \mathrm{~d} s=\int_{0}^{t} \int_{0}^{1} u_{t}\left(\rho^{1+\theta} u_{x}-\rho^{\gamma}\right)_{x} \mathrm{~d} x \mathrm{~d} s+\int_{0}^{t} \int_{0}^{1} u_{t} f \mathrm{~d} x \mathrm{~d} s
$$

Using integration by parts, (1.8) and the boundary conditions (1.10), we have

$$
\begin{aligned}
& \int_{0}^{t} \int_{0}^{1} u_{1}\left(\rho^{1+\theta} u_{x}-\rho^{\gamma}\right)_{x} \mathrm{~d} x \mathrm{~d} s=\int_{0}^{t} \int_{0}^{1} u_{t x}\left(\rho^{\gamma}-\rho^{1+\theta} u_{x}\right) \mathrm{d} x \mathrm{~d} s \\
& =\int_{0}^{1}\left\{u_{x}\left(\rho^{\gamma}-\frac{1}{2} \rho^{1+\theta} u_{x}\right)-u_{0 x}\left(\rho_{0}^{\gamma}-\frac{1}{2} \rho_{0}^{1+\theta} u_{0 x}\right)\right\} \mathrm{d} x \\
& +\int_{0}^{t} \int_{0}^{1}\left\{\gamma u_{x}^{2} \rho^{\gamma+1}-\frac{1+\theta}{2} u_{x}^{3} \rho^{2+\theta}\right\} \mathrm{d} x \mathrm{~d} s .
\end{aligned}
$$

Thus,

$$
\begin{aligned}
& \int_{0}^{t} \int_{0}^{1} u_{t}^{2} \mathrm{~d} x \mathrm{~d} s+\frac{1}{2} \int_{0}^{1} \rho^{1+\theta} u_{x}^{2} \mathrm{~d} x=\int_{0}^{1}\left\{u_{x} \rho^{\gamma}-u_{0 x}\left(\rho_{0}^{\gamma}-\frac{1}{2} \rho_{0}^{1+\theta} u_{0 x}\right)\right\} \mathrm{d} x \\
& +\int_{0}^{t} \int_{0}^{1}\left\{\gamma u_{x}^{2} \rho^{\gamma+1}-\frac{1+\theta}{2} u_{x}^{3} \rho^{2+\theta}\right\} \mathrm{d} x \mathrm{~d} s+\int_{0}^{t} \int_{0}^{1} u_{t} f \mathrm{~d} x \mathrm{~d} s \\
& \leq C_{1}(T)+\int_{0}^{1}\left(\frac{1}{4} \rho^{1+\theta} u_{x}^{2}+\rho^{2 \gamma-1-\theta}\right) \mathrm{d} x+C_{1}(T) \int_{0}^{t} \sup _{[0,1]}^{\gamma-\theta} \int_{0}^{1} \rho^{1+\theta} u_{x}^{2} \mathrm{~d} x \mathrm{~d} s \\
& +C_{1}(T) \int_{0}^{t} \int_{0}^{1} \rho^{1+\theta}\left|u_{x}\right|^{3} \mathrm{~d} x \mathrm{~d} s+\frac{1}{4} \int_{0}^{t} \int_{0}^{1} u_{t}^{2} \mathrm{~d} x \mathrm{~d} s+C_{1}(T) \int_{0}^{1} f_{0}^{2} \mathrm{~d} x \mathrm{~d} s .
\end{aligned}
$$

Using Lemmas 2.1-2.2, we derive

$$
\int_{0}^{1} u_{x}^{2} \mathrm{~d} x+\int_{0}^{t} \int_{0}^{1} u_{t}^{2} \mathrm{~d} x \mathrm{~d} s \leq C_{1}(T)+C_{1}(T) \int_{0}^{t} \int_{0}^{1} \rho^{1+\theta}\left|u_{x}\right|^{3} \mathrm{~d} x \mathrm{~d} s
$$


The last term on the right-hand side of (2.21) can be estimated as follows, using (1.8), conditions (1.10) and Lemmas 2.1-2.2,

$$
\begin{aligned}
& C_{1}(T) \int_{0}^{t} \int_{0}^{1} \rho^{1+\theta}\left|u_{x}\right|^{3} \mathrm{~d} x \mathrm{~d} s \\
\leq & C_{1}(T) \int_{0}^{t} \max _{[0,1]}\left|\rho^{1+\theta} u_{x}\right| u_{x}^{2} \mathrm{~d} x \mathrm{~d} s \\
\leq & C_{1}(T) \int_{0}^{t} \max _{[0,1]}\left|\rho^{1+\theta} u_{x}-\rho^{\gamma}\right| \int_{0}^{1} u_{x}^{2} \mathrm{~d} x \mathrm{~d} s+C_{1}(T) \int_{0}^{t} \int_{0}^{1} u_{x}^{2} \mathrm{~d} x \mathrm{~d} s \\
\leq & C_{1}(T)+C_{1}(T) \int_{0}^{t} \int_{0}^{1} \mid\left(\rho^{1+\theta} u_{x}-\rho^{\gamma}\right)_{x} \mathrm{~d} s \int_{0}^{1} u_{x}^{2} \mathrm{~d} x \mathrm{~d} s \\
\leq & C_{1}(T)+C_{1}(T) \int_{0}^{t} \int_{0}^{1}\left|u_{t}-\mathrm{d} s \int_{0}^{1} u_{x}^{2} \mathrm{~d} x \mathrm{~d} s+C_{1}(T) \int_{0}^{t} \int_{0}^{1}\right| f \mid \mathrm{d} s \int_{0}^{1} u_{x}^{2} \mathrm{~d} x \mathrm{~d} s \\
\leq & C_{1}(T)+\frac{1}{4} \int_{0}^{t} \int_{0}^{1} u_{t}^{2} \mathrm{~d} x \mathrm{~d} s+C_{1}(T) \int_{0}^{t} \int_{0}^{1} f^{2} \mathrm{~d} x \mathrm{~d} s+C_{1}(T) \int_{0}^{1}\left(\int_{0}^{t} u_{x}^{2} \mathrm{~d} x\right)^{2} \mathrm{~d} s \\
\leq & C_{1}(T)+\frac{1}{4} \int_{0}^{t} \int_{0}^{1} u_{t}^{2} \mathrm{~d} x \mathrm{~d} s+C_{1}(T) \int_{0}^{1}\left(\int_{0}^{2} u_{x}^{2} \mathrm{~d} x\right)^{\mathrm{d} s} .
\end{aligned}
$$

Inserting the above estimate into (2.21),

$$
\int_{0}^{1} u_{x}^{2} \mathrm{~d} x+\int_{0}^{t} \int_{0}^{1} u_{t}^{2} \mathrm{~d} x \mathrm{~d} s \leq C_{1}(T)+C \int_{0}^{t}\left\|u_{x}\right\|^{2} \int_{0}^{1} u_{x}^{2} \mathrm{~d} x \mathrm{~d} s .
$$

which, by virtue of Gronwall's inequality, (2.1) and (2.14), gives (2.19).

Proof of Theorem 2.1 By Lemmas 2.1-2.3, we complete the proof of Theorem 2.1.

\section{Global existence of solutions in $\boldsymbol{H}^{2}$}

For external force $f(r, t)$, we suppose

$$
f(r, t) \in L^{\infty}\left([0, T], L^{2}[0,1]\right), f_{r}(r, t) \in L^{2}\left([0, T], L^{2}[0,1]\right), f_{t}(r, t) \in L^{2}\left([0, T], L^{2}[0,1]\right)
$$

Constant $C_{2}(T)$ denotes generic positive constant depending only on the $\mathrm{H}^{2}$-norm of initial data $\left(\rho_{0}, u_{0}\right),\|f\|_{\left.L^{\infty}([0, T]), L^{2}[0,1]\right)},\left\|f_{r}\right\|_{L^{2}\left([0, T], L^{2}[0,1]\right)}\left\|f_{t}\right\|_{L^{2}\left([0, T], L^{2}[0,1]\right)}$, time $T$ and constant $C_{1}(T)$.

Remark 3.1 By (3.1), we easily know that assumptions (3.1) is equivalent to the following conditions

$$
\begin{aligned}
& f(r(x, t), t) \in L^{\infty}\left([0, T], L^{2}[0,1]\right), \\
& f_{r}(r(x, t), t) \in L^{2}\left([0, T], L^{2}[0,1]\right), f_{t}(r(x, t), t) \in L^{2}\left([0, T], L^{2}[0,1]\right) .
\end{aligned}
$$

Therefore, the generic constant $C_{2}(T)$ depending only on the norm of initial data $\left(\rho_{0}\right.$, $\left.u_{0}\right)$ in $H^{2}$, the norms of $f$ in the class of functions in (3.2)-(3.3) and time $T$. 
Theorem 3.1 Let $0<\theta<1, \gamma>1$, and assume that the initial data satisfies $\left(\rho_{0}, u_{0}\right) \in$ $H^{2}$ and external force $f$ satisfies conditions (3.1), then there exists a unique global solution $(\rho(x, t), u(x, t))$ to problem (1.8)-(1.11), such that for any $T>0$,

$$
\begin{aligned}
& \rho \in L^{\infty}\left([0, T], H^{2}[0,1]\right), \quad u \in L^{\infty}\left([0, T], H^{2}[0,1] \cap \in L^{2}\left([0, T], H^{3}[0,1]\right),\right. \\
& u_{t} \in L^{\infty}\left([0, T], L^{2}[0,1]\right) \cap \in L^{2}\left([0, T], H^{1}[0,1]\right) .
\end{aligned}
$$

The proof of Theorem 3.1 can be divided into the following several lemmas.

Lemma 3.2 Under the assumptions in Theorem 3.1, for any $0 \leq t \leq T$, we have the following estimates

$$
\begin{aligned}
& \left\|u_{t}(t)\right\|^{2}+\int_{0}^{t} \int_{0}^{1} u_{t x}^{2}(x, s) \mathrm{d} x \mathrm{~d} s \leq C_{2}(T), \\
& \left\|u_{x}(t)\right\|_{L}^{2} \infty+\left\|u_{x x}(t)\right\|^{2} \mathrm{~d} x \leq C_{2}(T) .
\end{aligned}
$$

Proof Differentiating (1.9) with respect to $t$, multiplying the resulting equation by $u_{t}$ in $L^{2}[0,1]$, performing an integration by parts, and using Lemma 2.1 , we have

$$
\begin{gathered}
\frac{1}{2} \frac{\mathrm{d}}{\mathrm{d} t}\left\|u_{t}\right\|^{2}+\int_{0}^{1} \rho^{1+\theta} u_{t x}^{2} \mathrm{~d} x=\int_{0}^{1}\left((\theta+1) \rho^{\theta+2} u_{x}^{2}-\gamma \rho^{\gamma+1} u_{x}+\frac{\partial f}{\partial t}\right) u_{t x} \mathrm{~d} x \\
\leq \frac{1}{2} \int_{0}^{1} \rho^{1+\theta} u_{t x}^{2} \mathrm{~d} x+C_{1}(T) \int_{0}^{1}\left(\rho^{2 \theta+3} u_{x}^{4}+\rho^{2 \gamma+1-\theta} u_{x}^{2}\right) \mathrm{d} x \\
+C_{1}(T) \int_{0}^{1}\left(\left(f_{r} r_{t}\right)^{2}+f_{t}^{2}\right) \mathrm{d} x .
\end{gathered}
$$

Integrating (3.8) with respect to $t$, applying the interpolation inequality, we conclude

$$
\begin{aligned}
& \left\|u_{t}(t)\right\|^{2}+\int_{0}^{t} \int_{0}^{1} \rho^{1+\theta} u_{t x}^{2} \mathrm{~d} x \mathrm{~d} s \\
& \leq\left\|u_{t}(x, 0)\right\|+C_{1}(T) \int_{0}^{t} \int_{0}^{1}\left(u_{x}^{4}+u_{x}^{2}+f_{r}^{2} u^{2}+f_{t}^{2}\right) \mathrm{d} x \mathrm{~d} s \\
& \leq\left\|u_{t}(x, 0)\right\|+C_{1}(T) \int_{0}^{t}\left[u_{x}^{2}+\left(\left\|u_{x x}\right\|^{\frac{1}{4}}\left\|u_{x}\right\|^{\frac{3}{4}}+\left\|u_{x}\right\|^{4}\right)(s)\right] \mathrm{d} s \\
& \quad+\int_{0}^{t}\|u\|_{L^{\infty}}^{2} \int_{0}^{1} f_{r}^{2} \mathrm{~d} x \mathrm{~d} s+C_{1}(t) \int_{0}^{t} \int_{0}^{1} f_{t}^{2} \mathrm{~d} x \mathrm{~d} s .
\end{aligned}
$$

On the other hand, by (1.9), we get

$$
u_{0 t}=-\gamma \rho_{0}^{\gamma-1} \rho 0_{x}+\rho_{0}^{\theta+1} u 0_{x x}+(\theta+1) \rho_{0}^{\theta} \rho 0_{x} u 0_{x}+f\left(r_{0}, 0\right) .
$$


We derive from assumption (3.1) and (3.10) that

$$
\int_{0}^{1} u_{0 t}^{2}(x) \mathrm{d} x \leq C_{2}(T)
$$

Inserting (3.11) into (3.9), by virtue of Lemmas 2.1-2.3 and assumption (3.1), we obtain (3.6). We infer from (1.9),

$$
u_{t}=-\gamma \rho^{\gamma-1} \rho_{x}+\rho^{\theta+1} u_{x x}+(\theta+1) \rho^{\theta} \rho_{x} u_{x}+f(r, t)
$$

Multiplying (3.12) by $u_{x x}$ in $L^{2}[0,1]$, we deduce

$$
\int_{0}^{1} \rho^{\theta+1} u_{x x}^{2} \mathrm{~d} x=\int_{0}^{1} u_{x x}\left(u_{t}+\gamma \rho^{\gamma-1} \rho_{x}-(\theta+1) \rho^{\theta} \rho_{x} u_{x}-f(r, t)\right) \mathrm{d} x .
$$

Using Young's inequality and Sobolev's embedding theorem $W^{1,1} \otimes W^{\infty}$, Lemma 2.1 and (3.6), we deduce from (3.13) that

$$
\begin{aligned}
\int_{0}^{1} u_{x x}^{2} \mathrm{~d} x & \leq C_{1}(T) \int_{0}^{1}\left(u_{t}^{2}+\rho_{x}^{2}+\rho_{x}^{2} u_{x}^{2}+f^{2}\right) \mathrm{d} x+\frac{1}{4} \int_{0}^{1} u_{x x}^{2} \mathrm{~d} x \\
& \leq C_{2}(T)+C_{1}(T)\left\|u_{x}\right\|_{L^{\infty}}^{2} \int_{0}^{1} \rho_{x}^{2} \mathrm{~d} x+\frac{1}{4} \int_{0}^{1} u_{x x}^{2} \mathrm{~d} x \\
& \leq C_{2}(T)+\frac{1}{2} \int_{0}^{1} u_{x x}^{2} \mathrm{~d} x
\end{aligned}
$$

whence

$$
\int_{0}^{1} u_{x x}^{2} d x \leq C_{2}(T)
$$

Applying embedding theorem, we derive from (3.14) that

$$
\left\|u_{x}\right\|_{L^{\infty}}^{2} \leq C_{1}(T)\left(\left\|u_{x}\right\|^{2}+\left\|u_{x x}\right\|^{2}\right) \leq C_{2}(T)
$$

which, along with (3.14), gives (3.7). The proof is complete.

Lemma 3.3 Under the assumptions in Theorem 3.1, for any $0 \leq t \leq T$, we have the following estimates

$$
\begin{aligned}
& \left\|\rho_{x x}(t)\right\|^{2}+\int_{0}^{t}\left\|\rho_{x x}(s)\right\|^{2} \mathrm{~d} s \leq C_{2}(T), \\
& \int_{0}^{t}\left\|u_{x x x}(s)\right\|^{2} \mathrm{~d} x \leq C_{2}(T) .
\end{aligned}
$$


Proof Differentiating (1.9) with respect to $x$, exploiting (1.8), we have

$$
\begin{aligned}
u_{t x}= & \left(-\rho^{\gamma}+\rho^{1+\theta} u_{x}\right)_{x x}+\frac{\mathrm{d} f}{\mathrm{~d} x} \\
= & -\gamma(\gamma-1) \rho^{\gamma-2} \rho_{x}^{2}-\gamma \rho^{\gamma-1} \rho_{x x}+(\theta+1) \theta \rho^{\theta-1} \rho_{x}^{2} u_{x} \\
& +(\theta+1) \rho^{\theta} \rho_{x x} u_{x}+2(\theta+1) \rho^{\theta} \rho_{x} u_{x x}+\rho^{\theta+1} u_{x x x}+\frac{1}{\rho} f_{r}
\end{aligned}
$$

which gives

$$
\left(\rho^{\theta-1} \rho_{x x}\right)_{t}+P_{\rho} \rho_{x x}=E(x, t),
$$

with

$$
E(x, t)=-P_{\rho \rho} \rho_{x}^{2}-2(1-\theta) \rho^{\theta} \rho_{x} u_{x x}+(1+\theta) \theta \rho^{\theta-1} \rho_{x}^{2} u_{x}-2 \rho^{\theta-1} \rho_{x}^{2} u_{x}-u_{t x}+\frac{1}{\rho} f_{r} .
$$

Multiplying (3.18) by $\rho^{\theta-1} \rho_{x x}$, integrating the resultant over $[0,1]$, using condition (3.1), Young's inequality, Lemma 3.2 and Theorem 2.1, we deduce

$$
\frac{\mathrm{d}}{\mathrm{d} t}\left\|\rho^{\theta-1} \rho_{x x}\right\|^{2}+\int_{0}^{1} \gamma \rho^{\gamma+\theta-2} \rho_{x x}^{2} \mathrm{~d} x \leq C_{1}(T) \int_{0}^{1}\left(\rho_{x}^{4}+u_{t x}^{2}+\rho_{x}^{4} u_{x}^{2}+\rho_{x}^{2} u_{x x}^{2}+f_{x}^{2}\right) \mathrm{d}
$$

Integrating (3.19) with respect to $t$ over $[0, \mathrm{t}]$, using Theorem 2.1, Lemma 3.2 and the interpolation inequality, we derive

$$
\begin{aligned}
& \left\|\rho_{x x}(t)\right\|^{2}+\int_{0}^{t}\left\|\rho_{x x}(s)\right\|^{2} \mathrm{~d} s \\
& \leq C_{2}(T)+C_{1}(T) \int_{0}^{t}\left\|u_{x}\right\|_{L^{\infty}}^{2} \int_{0}^{1} \rho_{x}^{2} \mathrm{~d} x \mathrm{~d} s+C_{1}(T) \int_{0}^{t} \int_{0}^{1}\left(\rho_{x}^{4}+u_{t x}^{2}\right) \mathrm{d} x \mathrm{~d} s \\
& +C_{1}(T) \int_{0}^{t}\left\|\rho_{x}\right\|_{L^{\infty}}^{2} \int_{0}^{1} u_{x x}^{2} \mathrm{~d} x \mathrm{~d} s+C_{1}(T) \int_{0}^{t} \int_{0}^{1} f_{r}^{2} \mathrm{~d} x \mathrm{~d} s \\
& \leq C_{2}(T)+C_{1}(T) \int_{0}^{t} \int_{0}^{1} \rho_{x}^{2} \mathrm{~d} x \mathrm{~d} s+\frac{1}{2} \int_{0}^{t}\left\|\rho_{x x}(s)\right\|^{2} \mathrm{~d} s
\end{aligned}
$$

which, along with Lemma 2.1, gives estimate (3.15).

Differentiating (1.9) with respect to $x$, we can obtain

$$
\begin{aligned}
u_{x x x} & =\rho^{-1-\theta}\left(u_{t x}+\gamma(\gamma-1)\right) \rho^{\gamma-2} \rho_{x}^{2}+\gamma \rho^{\gamma-1} \rho_{x x} \\
& \left.-\left((\theta+1) \rho^{\theta} \rho_{x x} u_{x}+2(\theta+1) \rho^{\theta} \rho_{x} u_{x x}+(\theta+1) \theta \rho^{\theta-1} \rho_{x}^{2} u_{x}\right)-\frac{\partial f}{\partial x}\right) .
\end{aligned}
$$


Integrating (3.21) with respect to $x$ and $t$ over $[0,1] \times[0, t]$, applying the embedding theorem, Lemmas 2.1-2.3 and Lemma 3.1, and the estimate (3.15), we conclude

$$
\begin{aligned}
\int_{0}^{t} \int_{0}^{1} u_{x x x}^{2} \mathrm{~d} x \mathrm{~d} s & \leq C_{1}(T) \int_{0}^{t} \int_{0}^{1}\left(u_{t x}^{2}+\rho_{x}^{4}+\rho_{x x}^{2}+\rho_{x}^{2} u_{x x}^{2}+\rho_{x}^{4} u_{x}^{2}+\rho_{x x}^{2} u_{x}^{2}+f_{r}^{2}\right) \mathrm{d} x \mathrm{~d} s \\
\leq & C_{1}(T) \int_{0}^{t}\left\|u_{x}\right\|_{L^{\infty}}^{2} \int_{0}^{1}\left(\rho_{x x}^{2}+\rho_{x}^{4}\right) \mathrm{d} x \mathrm{~d} s+C_{1}(T) \int_{0}^{t}\left\|\rho_{x}\right\|_{L^{\infty}}^{2}\left\|u_{x x}\right\|^{2} \mathrm{~d} s \\
& +C_{1}(T) \int_{0}^{t} \int_{0}^{1}\left(\rho_{x}^{4}+u_{t x}^{2}+\rho_{x x}^{2}+f_{r}^{2}\right) \mathrm{d} x \mathrm{~d} s \\
\leq & C_{2}(T) .
\end{aligned}
$$

The proof is complete.

Proof of Theorem 3.1 By Lemmas 3.2-3.3, Theorem 2.1 and Sobolev's embedding theorem, we complete the proof of Theorem 3.1.

\section{Global existence of solutions in $H^{4}$}

For external force $f(r, t)$, besides (3.1), we assume that

$$
f_{r}, f_{t}, f_{r t} \in L^{\infty}\left([0, T], L^{2}[0,1]\right), \quad f_{r r}, f_{r t}, f_{t t}, f_{r r r} \in L^{2}\left([0, T], L^{2}[0,1]\right) .
$$

Remark 4.1 By (4.1), we easily know that assumptions (4.1) is equivalent to the following conditions

$$
\begin{aligned}
& f_{r}(r(x, t), t), f_{t}(r(x, t), t), f_{r r}(r(x, t), t) \in L^{\infty}\left([0, T], L^{2}[0,1]\right), \\
& f_{r r}(r(x, t), t), f_{r t}(r(x, t), t), f_{t t}(r(x, t), t), f_{r r r}(r(x, t), t) \in L^{2}\left([0, T], L^{2}[0,1]\right) .
\end{aligned}
$$

Therefore, the generic constant $C_{4}(T)$ depending only on the norm of initial data $\left(\rho_{0}\right.$, $\left.u_{0}\right)$ in $H^{4}$, the norms of $f$ in the class of functions in (4.2)-(4.3) and time $T$.

Theorem 4.1 Let $0<\theta<1, \gamma>1$, and assume that the initial data satisfies $\left(\rho_{0}, u_{0}\right) \in$ $H^{4}$ and external force $f$ satisfies conditions (4.1), then there exists a unique global solution $(\rho(x, t), u(x, t))$ to problem (1.8)-(1.11), such that for any $T>0$,

$$
\begin{aligned}
& \rho \in L^{\infty}\left([0, T], H^{4}[0,1]\right), \quad \rho_{t} \in L^{\infty}\left([0, T], H^{3}[0,1]\right) \cap \in L^{2}\left([0, T], H^{4}[0,1]\right), \\
& \rho_{t t} \in \in L^{\infty}\left([0, T], H^{1}[0,1]\right) \cap \in L^{2}\left([0, T], H^{2}[0,1]\right) \\
& u \in L^{\infty}\left([0, T], H^{4}[0,1]\right) \cap \in L^{2}\left([0, T], H^{5}[0,1]\right) \\
& u_{t} \in L^{\infty}\left([0, T], H^{2}[0,1]\right) \cap \in L^{2}\left([0, T], H^{3}[0,1]\right) \\
& u_{t t} \in L^{\infty}\left([0, T], L^{2}[0,1]\right) \cap \in L^{2}\left([0, T], H^{1}[0,1]\right)
\end{aligned}
$$

The proof of Theorem 4.1 can be divided into the following several lemmas. 
Lemma 4.2 Under the assumptions of Theorem 4.1, the following estimates hold for any $t \in[0, T]$,

$$
\begin{aligned}
& \left\|u_{t x}(x, 0)\right\|+\left\|u_{t x x}(x, 0)\right\|+\left\|u_{t t}(x, 0)\right\| \leq C_{4}(T), \\
& \left\|u_{t t}(t)\right\|^{2}+\int_{0}^{t}\left\|u_{t t x}(s)\right\|^{2} \mathrm{~d} s \leq C_{4}(T)
\end{aligned}
$$

Proof We easily infer from (1.9) and Theorem 2.1, Theorem 3.1 that

$$
\left\|u_{t}(t)\right\| \leq C_{2}(T)\left(\left\|u_{x}(t)\right\|_{H^{1}}+\left\|\rho_{x}(t)\right\|+\|f(t)\|\right) .
$$

Differentiating (1.9) with respect to $x$ and exploiting Lemmas 2.1-2.3, we have

$$
\left\|u_{t x}(t)\right\| \leq C_{2}(T)\left(\left\|u_{x}(t)\right\|_{H^{2}}+\left\|\rho_{x}(t)\right\|_{H^{1}}+\left\|f_{r}(t)\right\|\right),
$$

or

$$
\left\|u_{x x x}(t)\right\| \leq C_{2}(T)\left(\left\|u_{x}(t)\right\|_{H^{1}}+\left\|\rho_{x}(t)\right\|_{H^{1}}+\left\|u_{t x}(t)\right\|+\left\|f_{r}(t)\right\|\right) .
$$

Differentiating (1.9) with respect to $x$ twice, using Lemmas 2.1-2.3, 3.2-3.3 and the embedding theorem, we have

$$
\left\|u_{t x x}(t)\right\| \leq C_{2}(T)\left(\left\|u_{x}(t)\right\|_{H^{3}}+\left\|\rho_{x}(t)\right\|_{H^{2}}+\left\|f_{r}(t)\right\|+\left\|f_{r r}(t)\right\|\right),
$$

or

$$
\left\|u_{x x x x}(t)\right\| \leq C_{2}(T)\left(\left\|u_{x}(t)\right\|_{H^{2}}+\left\|\rho_{x}(t)\right\|_{H^{2}}+\left\|u_{t x x}(t)\right\|+\left\|f_{r}(t)\right\|+\left\|f_{r r}(t)\right\|\right) .
$$

Differentiating (1.9) with respect to $t$, and using Lemmas 2.1-2.3 and (1.8), we deduce that

$$
\left\|u_{t t}(t)\right\| \leq C_{2}(T)\left(\left\|u_{t x}(t)\right\|+\left\|u_{x}(t)\right\|_{H^{1}}+\left\|\rho_{x}(t)\right\|+\left\|u_{t x x}(t)\right\|+\left\|f_{r}(t)\right\|+\left\|f_{t}(t)\right\|\right)
$$

which together with (4.12) and (4.14) implies

$$
\left\|u_{t t}(t)\right\| \leq C_{2}(T)\left(\left\|u_{x}(t)\right\|_{H^{3}}+\left\|\rho_{x}(t)\right\|_{H^{2}}+\left\|f_{r}(t)\right\|+\left\|f_{t}(t)\right\|+\left\|f_{r r}(t)\right\|\right) .
$$

Thus, estimate (4.9) follows from (4.12), (4.14), (4.17) and condition (4.1).

Now differentiating (1.9) with respect to $t$ twice, multiplying the resulting equation by $u_{t t}$ in $L^{2}([0,1])$, and using integration by parts, (1.8) and the boundary condition (1.10), we deduce

$$
\begin{aligned}
& \int_{0}^{1} u_{t t t} u_{t t} \mathrm{~d} x=\int_{0}^{1}\left[\left(-\rho^{\gamma}+\rho^{1+\theta} u_{x}\right)_{t t x}+\frac{\mathrm{d}^{2} f}{\mathrm{~d} t^{2}}\right] u_{t t} \mathrm{~d} x \\
& =-\int_{0}^{1}\left(-\rho^{\gamma}+\rho^{1+\theta} u_{x}\right)_{t t} u_{t t x} \mathrm{~d} x+\int_{0}^{1}\left(f_{r r} r_{t}^{2}+f_{r} r_{t t}+f_{r t}+f_{r t} r_{t}+f_{t t}\right) u_{t t} \mathrm{~d} x \\
& \leq-\int_{0}^{1} \rho^{1+\theta} u_{t t x}^{2} \mathrm{~d} x+\frac{1}{2} \int_{0}^{1} \rho^{1+\theta} u_{t t x}^{2} \mathrm{~d} x+\frac{1}{2} \int_{0}^{1} u_{t t}^{2} \mathrm{~d} x \\
& +C_{1}(T) \int_{0}^{1}\left(u_{x}^{4}+u_{t x}^{2}+u_{x}^{2} u_{t x}^{2}+u_{x}^{6}+f_{r}^{2} u_{t}^{2}+f_{r r}^{2}+f_{t}^{2}+f_{t t}^{2}\right) \mathrm{d} x
\end{aligned}
$$


here, we use $\frac{d^{2} f}{d t^{2}}=f_{r r} r_{t}^{2}+f_{r t} r_{t}+f_{r} r_{1}^{2}+f_{t t}$. Integrating (4.18) with respect to $t$, applying assumption (4.1) and (4.9), we have

$$
\begin{aligned}
& \left\|u_{t t}(t)\right\|^{2}+\int_{0}^{t} \int_{0}^{1} \rho^{1+\theta} u_{t t x}^{2} \mathrm{~d} x \mathrm{~d} s \\
& \leq C_{4}(T)+\frac{1}{2} \int_{0}^{t}\left\|u_{t t}(s)\right\|^{2} \mathrm{~d} s+C_{1}(T) \int_{0}^{t}\left(\left\|u_{x}\right\|^{2}+\left\|u_{t x}\right\|^{2}+\left\|u_{x}\right\|_{L^{6}}^{6}\right. \\
& \left.+\left\|u_{x}\right\|_{L^{\infty}}^{2}\left\|u_{t x}\right\|^{2}+\left\|f_{r}\right\|^{2}\left\|u_{t}\right\|_{L^{\infty}}^{2}\right)(s) \mathrm{d} s \\
& \leq C_{4}(T)+\frac{1}{2} \int_{0}^{t}\left\|u_{t t}(s)\right\|^{2} \mathrm{~d} s+C_{2}(T) \int_{0}^{t}\left(\left\|u_{x}\right\|_{H^{1}}^{2}+\left\|u_{t}\right\|_{H^{1}}^{2}\right)(s) \mathrm{d} s
\end{aligned}
$$

which, with Lemmas 2.1-2.3 and Theorem 3.1, implies

$$
\left\|u_{t t}(t)\right\|^{2}+\int_{0}^{t} \int_{0}^{1} \rho^{1+\theta} u_{t t x}^{2} \mathrm{~d} x \mathrm{~d} s \leq C_{4}(T)+\frac{1}{2} \int_{0}^{t}\left\|u_{t t}(s)\right\|^{2} \mathrm{~d} s, \quad \forall t \in[0, T] .
$$

If we apply Gronwall's inequality to (4.19), we conclude (4.11). The proof is complete.

Lemma 4.3 Under the assumptions of Theorem 4.1, the following estimate holds for any $t \in[0, T]$,

$$
\left\|u_{t x}(t)\right\|^{2}+\int_{0}^{t}\left\|u_{t x x}(s)\right\|^{2} \mathrm{~d} s \leq C_{4}(T) .
$$

Proof Differentiating (1.9) with respect to $x$ and $t$, multiplying the resulting equation by $u_{t x}$ in $L^{2}[0,1]$, and integrating by parts, we deduce that

$$
\begin{aligned}
& \int_{0}^{1} u_{t t x} u_{t x} \mathrm{~d} x=\int_{0}^{1}\left(\left(-\rho^{\gamma}+\rho^{1+\theta} u_{x}\right)_{t x x}+\frac{\partial^{2} f}{\partial t \partial_{x}}\right) u_{t x} \mathrm{~d} x \\
& =\left.\left(-\rho^{\gamma}+\rho^{1+\theta} u_{x}\right)_{t x} u_{t x}\right|_{0} ^{1}-\int_{0}^{1}\left(-\rho^{\gamma}+\rho^{1+\theta} u_{x}\right)_{t x} u_{t x x} \mathrm{~d} x \\
& \quad+\int_{0}^{1}\left(f_{r r} r_{t} r_{x}+f_{r} r_{t x}+f_{r t} r_{x}\right) u_{t x} \mathrm{~d} x \\
& =B_{1}+B_{2}+B_{3}
\end{aligned}
$$

where

$$
\begin{aligned}
& B_{1}=\left(-\rho^{\gamma}+\rho^{1+\theta} u_{x}\right)_{t x} u_{t x} x_{0}^{1} \quad B_{2}=-\int_{0}^{1}\left(-\rho^{\gamma}+\rho^{1+\theta} u_{x}\right)_{t x} u_{t x x} \mathrm{~d} x, \\
& B_{3}=\int_{0}^{1}\left(f_{r r} r_{t} r_{x}+f_{r} r_{t x}+f_{r t} r_{x}\right) u_{t x} \mathrm{~d} x .
\end{aligned}
$$


Employing Theorem 2.1, Theorem 3.1 Lemma 4.2 and the interpolation inequality, we conclude

$$
\begin{aligned}
B_{1} \leq & C_{2}(T)\left(\left\|u_{x x}\right\|_{L^{\infty}}+\left\|\rho_{x}\right\|_{L^{\infty}}\left\|u_{x}\right\|_{L^{\infty}}+\left\|\rho_{x}\right\|_{L^{\infty}}\left\|u_{t x}\right\|_{L^{\infty}}+\left\|u_{t x x}\right\|_{L^{\infty}}\right. \\
& \left.+\left\|u_{x}\right\|_{L^{\infty}}\left\|u_{x x}\right\|_{L^{\infty}}+\left\|\rho_{x}\right\|_{L^{\infty}}\left\|u_{x}\right\|_{L^{\infty}}^{2}\right)\left\|u_{t x}\right\|_{L^{\infty}} \\
\leq & C_{2}(T)\left(B_{01}+B_{02}\right)\left\|u_{t x}\right\| \frac{1}{2}\left\|u_{t x x}\right\| \frac{1}{2}
\end{aligned}
$$

with

$$
B_{01}=\left\|u_{x}\right\|_{H^{2}}+\left\|\rho_{x}\right\|_{H^{1}}, \quad B_{02}=\left\|u_{t x}\right\|^{\frac{1}{2}}|| u_{t x x}\left\|^{\frac{1}{2}}+\right\| u_{t x x}\left\|^{\frac{1}{2}}\right\| u_{t x x x} \|^{\frac{1}{2}} .
$$

Applying Young's inequality several times, we have that for any $\varepsilon \in(0,1)$,

$$
C_{2}(T) B_{01}|| u_{t x} \mid \frac{1}{2}\left\|u_{t x x}\right\|^{\frac{1}{2}} \leq \frac{\varepsilon^{2}}{2}\left\|u_{t x x}\right\|^{2}+C_{2}(T) \varepsilon^{-3}\left(\left\|u_{t x}\right\|^{2}+\left\|u_{x}\right\|_{H^{2}}^{2}=+\left\|\rho_{x}\right\|_{H^{1}}^{2}\right),
$$

and

$$
C_{2}(T) B_{02}\left\|u_{t x}\right\|^{\frac{1}{2}}\left\|u_{t x x}\right\|^{\frac{1}{2}} \leq \frac{\varepsilon^{2}}{2}\left\|u_{t x x}\right\|^{2}+\varepsilon^{2}\left\|u_{t x x x}\right\|^{2}+\left\|C_{2}(T) \varepsilon^{-6}\right\| u_{t x} \|^{2} .
$$

Thus we infer from (4.22)-(4.24) that

$$
B_{1} \leq \varepsilon^{2}\left(\left\|u_{t x x}\right\|^{2}+\left\|u_{t x x x}\right\|^{2}\right)+C_{2}(T) \varepsilon^{-6}\left(\left\|u_{t x}\right\|^{2}+\left\|u_{x}\right\|_{H^{2}}^{2}+\left\|\rho_{x}\right\|_{H^{1}}^{2}\right)
$$

which, together with Theorem 2.1, Theorem 3.1 and Lemma 4.2, implies

$$
\int_{0}^{t} B_{1}(s) \mathrm{d} s \leq C_{2}(T)+\varepsilon^{2} \int_{0}^{t}\left(\left\|u_{t x x}\right\|^{2}+\left\|u_{t x x x}\right\|^{2}\right)(s) \mathrm{d} s .
$$

On the other hand, differentiating (1.9) with respect to $x$ and $t$, and using Theorem 3.1 and Lemma 4.2, we derive

$$
\begin{aligned}
\left\|u_{t x x x}(t)\right\|^{2} \leq & C_{2}(T)\left(\left\|u_{x}\right\|_{H^{2}}^{2}+\left\|\rho_{x}\right\|_{H^{1}}^{2}+\left\|u_{t x}\right\|_{H^{1}}^{2}+\left\|u_{t t x}\right\|^{2}+\left\|\frac{\partial^{2} f}{\partial x \partial t}\right\|^{2}\right) \\
\leq & C_{2}(T)\left(\left\|u_{x}\right\|_{H^{2}}^{2}+\left\|\rho_{x}\right\|_{H^{1}}^{2}+\left\|u_{t x}\right\|_{H^{1}}^{2}+\left\|u_{t t x}\right\|^{2}\right. \\
& \left.+\left\|f_{r r}\right\|^{2}+\left\|f_{r}\right\|^{2}\left\|u_{x}\right\|_{L^{\infty}}^{2}+\left\|f_{r t}\right\|^{2}\right)
\end{aligned}
$$

Inserting (4.27) into (4.26), employing Theorem 2.1, Theorem 3.1 and Lemma 4.2, we conclude

$$
\int_{0}^{t} B_{1}(s) \mathrm{d} s \leq C_{4}(T)+\varepsilon^{2} \int_{0}^{t}\left\|u_{t x x}(s)\right\|^{2} \mathrm{~d} s .
$$

Similarly, by Theorem 2.1, Theorem 3.1, Lemma 4.2 and the embedding theorem, we get that for any $\varepsilon \in(0,1)$,

$$
\begin{aligned}
B_{2} \leq & -\int_{0}^{1} \rho^{1+\theta} u_{t x x}^{2} \mathrm{~d} x+\varepsilon \int_{0}^{1} \rho^{1+\theta} u_{t x x}^{2} \mathrm{~d} x \\
& +C_{2}(T) \int_{0}^{1}\left(\rho_{x}^{2} u_{x}^{4}+\rho_{x}^{2} u_{t x}^{2}+\rho_{x}^{2} u_{x}^{2}+u_{x x}^{2}\right) \mathrm{d} x .
\end{aligned}
$$


By virtue of assumption (4.1), Theorem 2.1 and Theorem 3.1, we derive that

$$
\begin{aligned}
B_{3} & =C_{1}(T)\left(\|u\|_{L^{\infty}}\left\|f_{r r}\right\|+\left\|u_{x}\right\|_{L^{\infty}}\left\|f_{r}\right\|+\left\|f_{r t}\right\|\right) \\
& \leq C_{2}(T)\left(\left\|f_{r r}\right\|+\left\|f_{r}\right\|+\left\|f_{r t}\right\|\right)
\end{aligned}
$$

which, combined with (4.21) and (4.27)-(4.29), gives

$$
\begin{aligned}
& \frac{\mathrm{d}}{\mathrm{dt}}\left\|u_{t x}(t)\right\|^{2}+\int_{0}^{1} \rho^{1+\theta} u_{t x x}^{2} d x \leq \varepsilon^{2}\left(\left\|u_{t x x}\right\|^{2}+\left\|u_{t x x x}\right\|^{2}\right) \\
& \quad+C_{2}(T)\left(\left\|u_{t x}\right\|^{2}+\left\|u_{x}\right\|_{H^{2}}^{2}+\left\|\rho_{x}\right\|_{H^{1}}^{2}+\left\|f_{r r}\right\|^{2}+\left\|f_{r}\right\|^{2}+\left\|f_{t t}\right\|^{2}\right) .
\end{aligned}
$$

Integrating (4.30) with respect to $t$, picking $\varepsilon$ small enough, using Theorem 2.1 and Theorem 3.1, Lemma 4.2 and assumption (4.1), we complete the proof of estimate (4.20).

Lemma 4.4 Under the assumptions of Theorem 4.1, the following estimates hold for any $t \in[0, T]$,

$$
\begin{aligned}
& \left\|\rho_{x x x}(t)\right\|^{2}+\left\|\rho_{x x x x}(t)\right\| \leq C_{4}(T) \\
& \left\|u_{x x x}(t)\right\|_{H^{1}}^{2}+\left\|u_{t x x}(t)\right\|^{2}+\int_{0}^{t}\left(\left\|u_{t t}\right\|_{H^{1}}^{2}+\left\|u_{t x x}\right\|_{H^{1}}^{2}\right)(s) \mathrm{d} s \leq C_{4}(T) \\
& \int_{0}^{t}\left\|u_{x x x x}(s)\right\|_{H^{1}}^{2} \mathrm{~d} s \leq C_{4}(T)
\end{aligned}
$$

Proof Differentiating (3.18) with respect to $x$, we have

$$
\left(\rho^{\theta-1} \rho_{x x x}\right)_{t}+P_{\rho} \rho_{x x x}=E_{1}(x, t)
$$

where

$$
E_{1}(x, t)=E_{x}(x, t)-P_{\rho \rho} \rho_{x} \rho_{x x}-(\theta-1)\left(\rho^{\theta-2} \rho_{x} \rho_{x x}\right)_{t} .
$$

An easy calculation with the interpolation inequality, Theorem 2.1 and Theorem 3.1, gives

$$
\begin{aligned}
\left\|E_{x}(t)\right\| \leq & C_{2}(T)\left(\left\|\rho_{x}(t)\right\|_{L^{6}}^{3}+\left\|\rho_{x} \rho_{x x}\right\|+\left\|\rho_{x} u_{x x x}\right\|+\left\|\rho_{x x} u_{x x}\right\|\right. \\
& \left.+\left\|\rho_{x}\right\|\left\|_{L^{\infty}}^{3}\right\| u_{x}\|+\| \rho_{x}\left\|_{L^{\infty}}^{2}\right\| u_{x x}\|+\| u_{t x x}\|++\| \rho_{x}\left\|_{L^{\infty}}\right\| f_{r} \mid+\left\|f_{r r}\right\|\right) \\
\leq & C_{2}(T)\left(\left\|\rho_{x}(t)\right\|_{H^{1}}+\left\|u_{x}(t)\right\|_{H^{2}}+\left\|u_{t x x}\right\|+\left\|f_{r}\right\|+\left\|f_{r r}\right\|\right),
\end{aligned}
$$

and

$$
\left\|E_{1}\right\| \leq C_{2}(T)\left(\left\|\rho_{x}(t)\right\|_{H^{1}}+\left\|u_{x}(t)\right\|_{H^{2}}+\left\|u_{t x x}(t)\right\|+\left\|f_{r}\right\|+\left\|f_{r r}\right\|\right) .
$$

By virtue of Theorem 2.1 and Theorem 3.1, we infer from (4.36)-(4.37), (4.20) and assumption (4.1) that

$$
\int_{0}^{t}\left\|E_{1}(s)\right\|^{2} \mathrm{~d} s \leq C_{4}(T), \quad \forall t \in[0, T] .
$$


Now multiplying (4.34) by $\rho^{\theta-1} \rho_{x x x}$ in $L^{2}[0,1]$, we obtain

$$
\frac{d}{\mathrm{~d} t}\left\|\rho^{\theta-1} \rho_{x x x}\right\|^{2}+\left\|\rho_{x x x}(t)\right\|^{2} \leq C_{1}(T)\left\|E_{1}(T)\right\|^{2} .
$$

Integrating (4.39) with respect to $t$, using Theorem 2.1 and Theorem 3.1, assumption (4.1) and (4.38), we can get

$$
\left\|\rho_{x x x}(t)\right\|^{2}+\int_{0}^{t}\left\|\rho_{x x x}(s)\right\|^{2} \mathrm{~d} s \leq C_{4}(T), \quad \forall t \in[0, T] .
$$

By virtue of Theorem 2.1 and Theorem 3.1, we infer from (4.10), (4.15) and (4.40) that

$$
\left\|u_{x x x}(t)\right\|^{2}+\int_{0}^{t}\left\|u_{x x x}(s)\right\|_{H^{1}}^{2} \mathrm{~d} s \leq C_{4}(T), \quad \forall t \in[0, T] .
$$

Differentiating (1.9) with respect to $t$, using Theorem 2.1 and Theorem 3.1 and Lemmas 4.2-4.3, we infer that for any $t \in[0, T]$,

$$
\left\|u_{t x x}(t)\right\| \leq C_{2}(T)\left\|u_{t t}(t)\right\|+C_{2}(T)\left(\left\|u_{x}(t)\right\|_{H^{1}}+\left\|u_{t x}(t)\right\|+\left\|\rho_{x}(t)\right\|\right) \leq C_{4}(T)(4.4
$$

which, combined with (4.15), (4.40) and (4.42), gives

$$
\left\|u_{x x x x}(t)\right\|^{2}+\int_{0}^{t}\left\|u_{t x x}(s)\right\|^{2} \mathrm{~d} s \leq C_{4}(T), \quad \forall t \in[0, T] .
$$

Differentiating (4.34) with respect to $x$, we see that

$$
\left(\rho^{\theta-1} \rho_{x x x x}\right)_{t}+P_{\rho} \rho_{x x x x}=E_{2}(x, t),
$$

with

$$
E_{2}(x, t)=E_{1 x}(x, t)-P_{\rho \rho} \rho_{x} \rho_{x x x}-(\theta-1)\left(\rho^{\theta-2} \rho_{x} \rho_{x x x}\right)_{t}
$$

and

$$
\left.E_{1 x}(x, t)=E_{x x}(x, t)-P_{\rho \rho} \rho_{x} \rho_{x x}\right)_{x}-(\theta-1)\left(\rho^{\theta-2} \rho_{x} \rho_{x x}\right)_{t x} .
$$

Using the embedding theorem, (1.8), Theorem 2.1, Theorem 3.1 and Lemmas 4.1-4.2, we can deduce that

$$
\begin{aligned}
& \left\|E_{x x}(t)\right\| \leq C_{4}(T)\left(\left\|u_{x}(t)\right\|_{H^{3}}+\left\|\rho_{x}(t)\right\| H_{H^{2}}+\left\|f_{r}\right\|+\left\|f_{r r}\right\|+\left\|f_{r r r}\right\|\right) \\
& \left\|E_{1 x}(t)\right\| \leq C_{4}(T)\left(\left\|u_{x}(t)\right\|_{H^{3}}+\left\|\rho_{x}(t)\right\|_{H^{2}}+\left\|u_{t x}(t)\right\|_{H^{2}}+\left\|f_{r}\right\|+\left\|f_{r r}\right\|+\left\|f_{r r r}\right\|\right) \\
& \left\|E_{2}(t)\right\| \leq C_{4}(T)\left(\left\|u_{x}(t)\right\|_{H^{3}}+\left\|\rho_{x}(t)\right\|_{H^{2}}+\left\|u_{t x}(t)\right\|_{H^{2}}+\left\|f_{r}\right\|+\left\|f_{r r}\right\|+\left\|f_{r r}\right\|\right) .
\end{aligned}
$$

Inserting (4.46) into (4.47), we have

$$
\begin{aligned}
\left\|E_{2}(t)\right\| \leq & C_{4}(T)\left(\left\|u_{x}(t)\right\|_{H^{3}}+\left\|\rho_{x}(t)\right\|_{H^{2}}+\left\|u_{t x}(t)\right\|_{H^{1}}\right) \\
& \left.+\left\|u_{t t x}(t)\right\|+\left\|f_{r}(t)\right\|+\left\|f_{r r}(t)\right\|+\left\|f_{r r r}(t)\right\|\right) .
\end{aligned}
$$


By virtue of Theorems 2.1, 3.1, Lemmas 4.2-4.3, we derive from (4.40)-(4.43) and assumption (4.1) that

$$
\int_{0}^{t}\left\|E_{2}(s)\right\|^{2} \mathrm{~d} s \leq C_{4}(T), \quad \forall t \in[0, T] .
$$

Multiplying (4.44) by $\rho^{\theta-1} \rho_{x x x x}$ in $L^{2}[0,1]$, we get

$$
\frac{\mathrm{d}}{\mathrm{dt}}\left\|\rho^{\theta-1} \rho_{x x x x}\right\|^{2}+\left\|\rho_{x x x x}(t)\right\|^{2} \leq C_{1}(T)\left\|E_{2}(t)\right\|^{2} .
$$

Integrating (4.50) with respect to $t$, using condition (4.1) and (4.49), we conclude

$$
\left\|\rho_{x x x x}(t)\right\|^{2}+\int_{0}^{t}\left\|\rho_{x x x x}(s)\right\|^{2} \mathrm{~d} s \leq C_{4}(T), \quad \forall t \in[0, T] .
$$

Differentiating (1.9) with respect to $x$ three times, using Theorems 2.1, 3.1, Lemmas 4.2-4.3 and the interpolation inequality, we infer

$$
\begin{aligned}
\left\|u_{x x x x x}(t)\right\| & \leq C_{4}(T)\left(\left\|u_{t x x x}(t)\right\|+\left\|u_{x}(t)\right\|_{H^{3}}+\left\|\rho_{x}(t)\right\|_{H^{3}}+\left\|f_{r}(t)\right\|\right. \\
& \left.+\left\|f_{r r}(t)\right\|+\left\|f_{r r r}(t)\right\|\right) .
\end{aligned}
$$

Thus we conclude from (1.8), (4.27), (4.41), (4.43), (4.51) and assumption (4.1) that

$$
\int_{0}^{t}\left(\left\|u_{x x x x x}\right\|^{2}+\left\|u_{t x x x}\right\|^{2}\right)(s) \mathrm{d} s \leq C_{4}(T), \quad \forall t \in[0, T] .
$$

Thus (4.31) follows from (4.40) and (4.51), we can derive estimate (4.32)-(4.33) from Theorem 2.1, Theorem 3.1, Lemmas 4.2-4.3, $(4.41),(4,43)$ and $(4.53)$. The proof is complete.

Proof of Theorem 4.1 Using (1.8), Theorem 2.1, 3.1 and Lemmas 4.2-4.4 and the proper interpolation inequality, we readily get estimate (4.4)-(4.8) and complete the proof from Theorem 4.1.

Corollary 4.5 Under assumptions of Theorem 4.1 and some suitable compatibility conditions, the global solution $(\rho(x, t), u(x, t))$ to problem (1.8)-(1.11) is the classical solution verifying

$$
\|\rho(t)\|_{C^{3+1 / 2}}+\|u(t)\|_{C^{3+1 / 2}} \leq C_{4}(T) .
$$

Proof By the embedding theorem, we easily prove the corollary from Theorem 4.1.

\section{Acknowledgements}

The work is in part supported by Doctoral Foundation of North China University of Water Sources and Electric Power (No. 201087), the Natural Science Foundation of Henan Province of China (No. 112300410040) and the NNSF of China (No. 11101145).

Authors' contributions

All authors contributed to each part of this work equally.

Competing interests

The authors declare that they have no competing interests.

Received: 14 June 2011 Accepted: 3 November 2011 Published: 3 November 2011 


\section{References}

1. Hoff, D, Serre, D: The failure of continuous dependence on initial data for the Navier- Stokes equations of compressible flow. SIMA J Appl Math. 51, 887-898 (1991). doi:10.1137/0151043

2. Fang, D, Zhang, T: Discontinuous solutions of the compressible Navier-Stokes equations with degenerate viscosity coefficient and vacuum. J Math Anal Appl. 318, 224-245 (2006). doi:10.1016/j.jmaa.2005.05.050

3. Jiang, S, Xin, Z, Zhang, P: Global weak solutions to 1D compressible isentropic Navier-stokes equations with densitydependent viscosity. Meth Appl Anal. 12, 239-252 (2005)

4. Liu, T, Xin, Z, Yang, T: Vacuum states of compressible flow. Discret Cont Dyn Syst. 4, 1-32 (1998)

5. Okada, M, Matušü-Nečasová, Š, Makino, T: Free boundary problem for the equation of one-dimensional motion of compressible gas with density-dependent viscosity. Ann Univ Ferrara Sez VII (N.S.). 48, 1-20 (2002)

6. Xin, Z, Yao, Z: The existence, uniqueness and regularity for one-dimensional compressible Navier-Stokes equations. (Preprint)

7. Yang, T, Yao, Z, Zhu, C: Compressible Navier-Stokes equations with degenerate viscosity coefficient and vacuum. Commun Partial Differ Equ. 26, 965-981 (2001). doi:10.1081/PDE-100002385

8. Qin, Y, Huang, L, Yao, Z: Regularity of 1D compressible isentropic Navier-Stokes equations with density-dependent viscosity. J Differ Equ. 245, 3956-3973 (2008). doi:10.1016/j.jde.2008.03.014

9. Qin, Y, Huang, L, Yao, Z: A remark on regularity of 1D compressible isentropic Navier- Stokes equations with densitydependent viscosity. J Math Anal Appl. 351, 497-508 (2009). doi:10.1016/j.jmaa.2008.10.044

10. Yang, T, Zhao, H: A vacuum problem for the one-dimensional compressible Navier-Stokes equations with densitydependent viscosity. J Differ Equ. 184, 163-184 (2002). doi:10.1006/jdeq.2001.4140

11. Yang, T, Zhu, C: Compressible Navier-Stokes equations with degenerate viscosity coefficient and vacuum. Commun Math Phys. 230, 329-363 (2002). doi:10.1007/s00220-002-0703-6

12. Vong, S, Yang, T, Zhu, C: Compressible Navier-Stokes equations with degenerate viscosity coefficient and vacuum(II). J Differ Equ. 192, 475-501 (2003). doi:10.1016/S0022-0396(03)00060-3

13. Fang, D, Zhang, T: Compressible Navier-Stokes equations with vacuum state in one dimension. Commun Pure App Anal. 3, 675-694 (2004)

14. Fang, D, Zhang, T: A note on compressible Navier-Stokes equations with vacuum state in one dimension. Nonlinear Anal. 58, 719-731 (2004). doi:10.1016/j.na.2004.05.016

15. Qin, Y, Huang, L, Deng, S, Ma, Z, Su, X, Yang, X: Interior regularity of the compressible Navier-Stokes equations with degenerate viscosity coefficient and vacuum. Discret Cont Dyn Syst Ser S. 2, 163-192 (2009)

16. Qin, Y, Zhao, Y: Global existence and asymptotic behavior of the compressible Navier- Stokes equations for a 1D isothermal viscous gas. Math Models Methods Appl Sci. 18, 1383-1408 (2008). doi:10.1142/S0218202508003078

17. Zhang, T, Fang, D: Global behavior of compressible Navier-Stokes equations with a degenerate viscosity coefficient. Arch Ration Mech Anal. 182, 223-253 (2006). doi:10.1007/s00205-006-0425-6

doi:10.1186/1687-2770-2011-43

Cite this article as: Huang and Lian: Global behavior of 1D compressible isentropic Navier-Stokes equations with a non-autonomous external force. Boundary Value Problems 2011 2011:43.

\section{Submit your manuscript to a SpringerOpen ${ }^{\circ}$ journal and benefit from:}

- Convenient online submission

Rigorous peer review

- Immediate publication on acceptance

- Open access: articles freely available online

- High visibility within the field

- Retaining the copyright to your article

Submit your next manuscript at $>$ springeropen.com 